\title{
CICLOSPORINA TÓPICA COMO TRATAMIENTO DEL PRÚRIGO ACTÍNICO OCULAR
}

\section{TOPICAL CYCLOSPORINE IN THE TREATMENT OF OCULAR ACTINIC PRURIGO}

\author{
ORTIZ-CASTILLO JV ${ }^{1}$, BOTO-DE-LOS-BUEIS A ${ }^{2}$, DE-LUCAS-LAGUNA R ${ }^{2}$, PASTOR-NIETO B ${ }^{1}$, \\ PELÁEZ-RESTREPO N ${ }^{1}$, FONSECA-SANDOMINGO A ${ }^{1}$
}

\begin{abstract}
RESUMEN
Caso clínico: Presentamos el caso de una mujer de 14 años latinoamericana, con una limbo-conjuntivitis severa. Habia sido diagnosticada de prúrigo actínico (PA) por el dermatólogo. Con el diagnóstico de PA ocular se inició tratamiento con colirio de ciclosporina A $2 \%$ con buena respuesta.

Discusión: El PA es una fotodermatosis idiopática que afecta principalmente a la población mestiza de Latino América. Los ojos se ven afectados en el $45 \%$ de los casos, como una limbo-conjuntivitis severa semejante a la atópica. A pesar de la escasa bibliografía existente, la ciclosporina tópica representa la mejor alternativa en el tratamiento del PA ocular.
\end{abstract}

Palabras clave: Prúrigo actínico, ciclosporina tópica, conjuntivitis, limbitis, fotodermatosis.

\begin{abstract}
Case report: A 14-year-old girl from Peru suffered severe limbitis and conjunctivitis. She also presented with clinical skin features diagnosed as actinic prurigo (AP). Her symptoms were successfully controlled with sustained topical therapy of $2 \%$ Cyclosporine A.

Discussion: AP is an idiopathic photodermatosis that affects mainly the hispanic population of Latin America. There are ocular signs of severe limbitis and conjunctivitis (like atopic keratoconjunctivitis) in $45 \%$ of cases. Literature on the subject is very limited and currently topical cyclosporine seems to be the best therapy available (Arch Soc Esp Oftalmol 2006; 81: 661-664).
\end{abstract}

Key words: Actinic prurigo, topical cyclosporine, conjunctivitis, limbitis, photodermatosis.

Recibido: 27/3/06. Aceptado: 20/11/06.

Servicio de Oftalmología. Hospital La Paz. Madrid. España.

1 Licenciado en Medicina.

2 Doctor en Medicina.

Comunicación presentada en el LXXX Congreso de la S.E.O. (Córdoba 2004).

Los autores manifiestan que no tienen interés comercial ni han recibido apoyo económico.

Correspondencia:

Ana Boto de los Bueis

$\mathrm{C} /$. O'Donnell $32,1^{\circ} \mathrm{B}$

28009 Madrid

España

E-mail: anaboto@wanadoo.es 


\section{INTRODUCCIÓN}

El prúrigo actínico (PA) es una fotodermatosis propia de países latinoamericanos cuyas manifestaciones oculares pueden simular una conjuntivitis atópica (1). Presentamos el caso de una niña con limbo conjuntivitis severa por PA.

\section{CASO CLÍNICO}

Mujer de 14 años, peruana, que presentaba en los últimos 6 meses episodios severos de enrojecimiento y fotofobia, sin respuesta a colirio de dexametasona (Colircusí Dexametasona ${ }^{\circledR}$ 0,1 \%, Alcon Cusi, España) y levocabastina (Bilina ${ }^{\circledR}$, Esteve, Barcelona, España). Refería padecer dermatitis y conjuntivitis atópica desde los siete años, y haber tenido un episodio de queratitis herpética en ojo izquierdo (OI) hacía 4 años y una úlcera corneal en ojo derecho (OD) hacía dos meses.

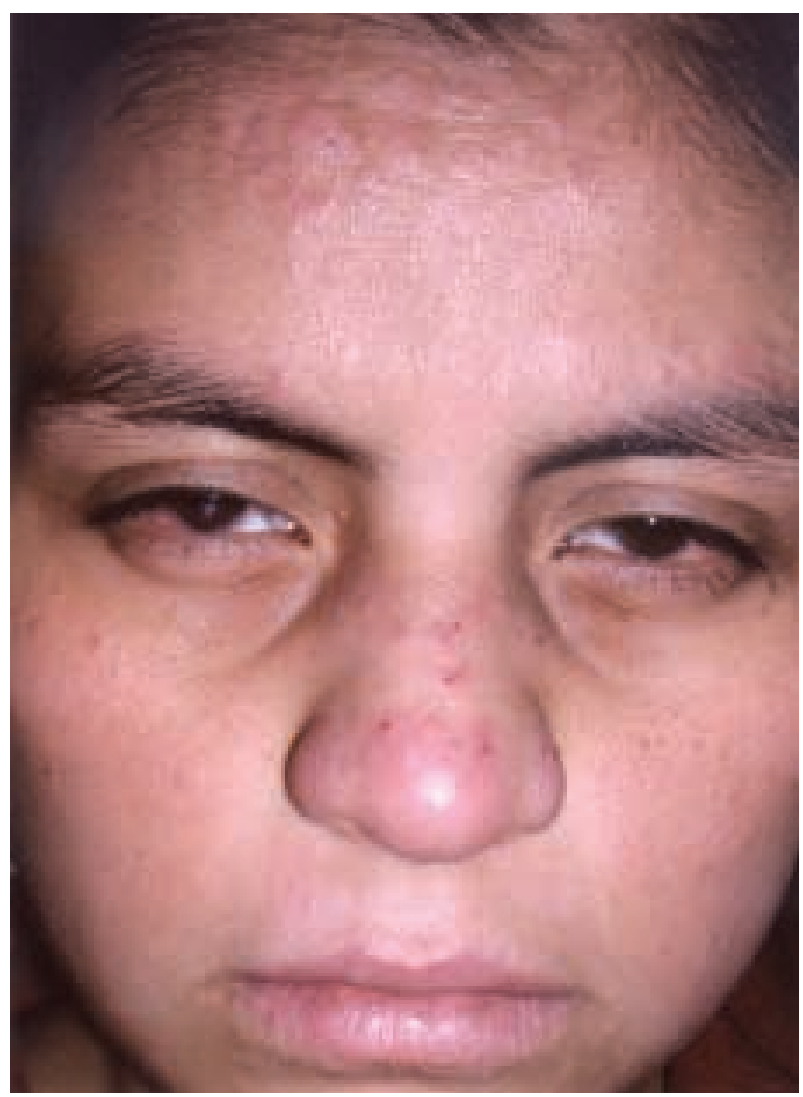

Fig. 1: Lesiones eritemato-descamativas faciales por prúrigo actínico.
La paciente presentaba lesiones cutáneas eritemato-descamativas en áreas malares, frontal, perilabial, dorso de la naríz, y dorso de las manos. No había afectación palpebral (fig. 1). En la exploración oftalmológica la agudeza visual corregida era CD a 2 mt en OD y 2/3 en OI, y en biomicroscopía presentaba en ambos ojos (AO) una reacción papilar en conjuntiva tarsal superior (fig. 2), hipertrofia limbar gelatinosa, parches de hiperpigmentación conjuntival bulbar (fig. 3) y queratitis punteada superficial. El OD presentaba pannus y un leucoma central, y el OI un pequeño leucoma paracentral.

No refería historia de atopia familiar, ni presentaba otras manifestaciones sistémicas de atopia. El estudio de alergia descartó sensibilización a alergenos comunes, la $\operatorname{IgE}$ sérica fue normal y $\operatorname{la} \operatorname{IgE}$ en lágrima normal (Lacrytest, laboratorio Adiatec,

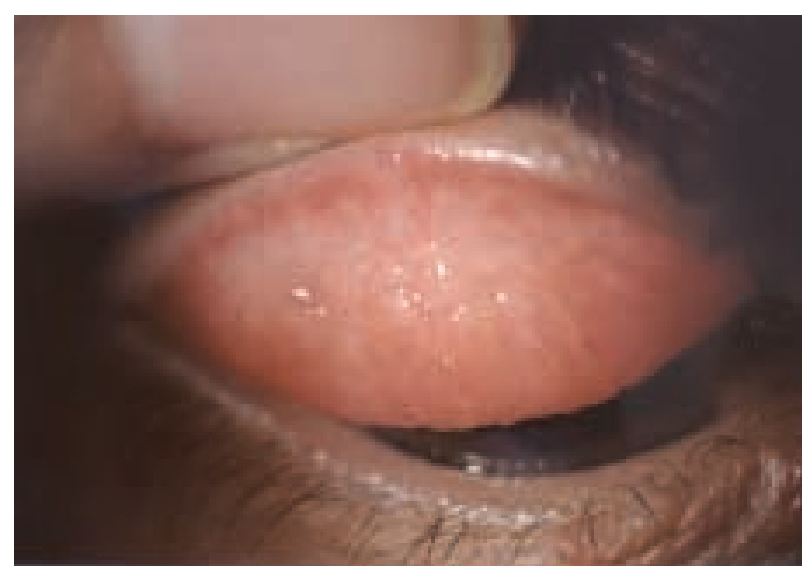

Fig. 2: Hipertrofia papilar en tarso superior de OD.

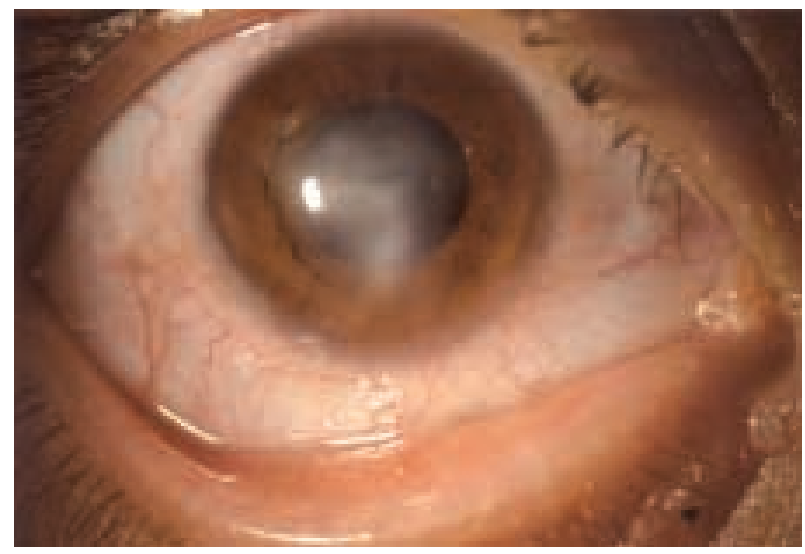

Fig. 3: Limbo-conjuntivitis OD por PA, y leucoma corneal central vascularizado secundario a úlcera infecciosa previa. 
Francia). Se consultó al servicio de dermatología que refirió haber sustituído el diagnóstico de dermatitis atópica hacía dos años por el de PA, tras el resultado de triple biopsia cutánea, comenzando entonces tratamiento tópico con pomada de tacrolimus $0,03 \%$ que ha mantenido hasta ahora, estabilizándose el cuadro cutáneo.

Con el diagnóstico de PA ocular, en septiembre de 2004 iniciamos tratamiento con colirio de ciclosporina $\mathrm{A}$ al $2 \%$, preparada en alcohol polivinílico por el servicio de farmacia, con caducidad de 28 días, a dosis inicial de 1 gota/8horas en $\mathrm{AO}$, con mejoría a las dos semanas de iniciado el tratamiento. En abril-05, y aún en tratamiento con colirio de ciclosporina $1 \% / 12 \mathrm{~h}$, presentó una reactivación del cuadro en $\mathrm{AO}$ que se controló aumentando el colirio a ciclosporina $2 \% / 8 \mathrm{~h}$. Una semana después presentó una dendrita herpética central en su OI que se resolvió con pomada de aciclovir (Zovirax pomada oftálmica ${ }^{\circledR}$, Glaxo-Wellcome, Tres Cantos, Madrid, España) 5 veces al día durante 21 días. Desde entonces mantiene como tratamiento colirio de ciclosporina $1 \% / 12 \mathrm{~h}$ y valaciclovir $500 \mathrm{mg} /$ día (Valtrex ${ }^{\circledR}$, GlaxoSmithKline, USA). En febrero de 2006 presenta una AV corregida OD de 1/8 y OI de $1 / 2$, y como único hallazgo inflamatorio leve limbitis temporal-inferior en AO.

\section{DISCUSIÓN}

Presentamos el caso de una mujer joven latinoamericana con un cuadro de limbo-conjuntivitis bilateral severa, atribuido inicialmente a atopia, y asociado a una dermatitis crónica, cuyo diagnóstico final fue de PA. El PA es una fotodermatosis idiopática crónica recidivante, causada por la reacción inmune de piel y mucosas a radiación UVA y UVB. Se presenta casi exclusivamente en países americanos que viven en altitudes superiores a $1.000 \mathrm{~m}$ sobre el nivel del mar, debutando entre los 6-8 años de edad. La piel se halla afectada en áreas foto expuestas como dorso de la nariz, malares y labios (2).

La clínica ocular se manifiesta en el $45 \%$ de los casos, afectando primariamente a conjuntiva y limbo, que muestran hiperemia difusa, secreción acuo- sa, áreas conjuntivales bulbares hiperpigmentadas, papilas tarsales hipertróficas, folículos, limbitis focal y pseudopterigium. El diagnóstico del PA está basado en la presentación clínica y el estudio anatomopatológico de mucosa labial, conjuntival o piel. La histología conjuntival tarsal muestra característicamente un adelgazamiento y atrofia del epitelio, vacuolización de células basales con infiltrados linfocitarios organizados en folículos submucosos, pigmentación conjuntival y eosinofilia, siendo el hallazgo histopatológico más característico del PA tanto en mucosa labial como en conjuntiva la presencia de folículos linfoides en la lámina propia (2).

La pomada de tacrolimus es la mejor alternativa para la dermatosis. Las lesiones oculares precisan de otro enfoque terapéutico. Actualmente, y a pesar de la escasa bibliografía al respecto, los estudios señalan a la ciclosporina tópica como la mejor opción en el tratamiento del prúrigo actínico ocular (3). En nuestro caso, la paciente mejoró con este tratamiento en aproximadamente 2 semanas, pero ha precisado mantenerlo para evitar recaídas. Aunque no hay experiencia en el empleo de tacrólimus tópico en el PA ocular, recientemente este colirio ha demostrado ser eficaz en casos de blefaroconjuntivitis crónicas (4) y conjuntivitis atópicas (5) severas. En un futuro, este macrólido inmunosupresor potente podría ser una alternativa terapéutica en casos de PA ocular resistentes al tratamiento con ciclosporina.

\section{BIBLIOGRAFÍA}

1. Magana M, Mendez Y, Rodriguez A, Mascott M. The conjunctivitis of solar (actinic) prurigo. Pediatr Dermatol 2000; 17: 432-435.

2. Hojyo-Tomoka T, Vega-Memije E, Granados J, Flores $O$, Cortes-Franco R, Teixeira F, et al. Actinic prurigo: an update. Int J Dermatol 1995; 34: 380-384.

3. McCoombes JA, Hirst LW, Green WR. Use of topical cyclosporin for conjunctival manifestations of actinic prurigo. Am J Ophthalmol 2000; 130: 830-831.

4. Joseph MA, Kaufman HE, Insler M. Topical tacrolimus ointment for treatment of refractory anterior segment inflammatory disorders. Cornea 2005; 24: 417-420.

5. Rikkers SM, Holland GN, Drayton GE, Michel FK, Torres $M F$, Takahashi S. Topical tacrolimus treatment of atopic eyelid disease. Am J Ophthalmol 2003; 135: 297-302. 